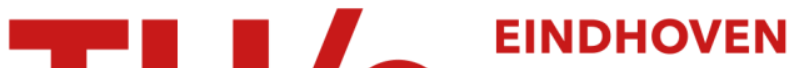 UNIVERSITY OF TECHNOLOGY
}

\section{Exploring the electron density in plasma induced by EUV radiation}

Citation for published version (APA):

Van Der Horst, R. M., Beckers, J., Osorio, E. A., Astakhov, D. I., Goedheer, W. J., Lee, C. J., Ivanov, V. V., Krivtsum, V. M., Koshelev, K. N., Lopaev, D. V., Bijkerk, F., \& Banine, V. Y. (2016). Exploring the electron density in plasma induced by EUV radiation: I. Experimental study in hydrogen. Journal of Physics D: Applied Physics, 49(14), 1-9. [145203]. https://doi.org/10.1088/0022-3727/49/14/145203

\section{Document license:}

TAVERNE

DOI:

10.1088/0022-3727/49/14/145203

Document status and date:

Published: 10/03/2016

\section{Document Version:}

Publisher's PDF, also known as Version of Record (includes final page, issue and volume numbers)

\section{Please check the document version of this publication:}

- A submitted manuscript is the version of the article upon submission and before peer-review. There can be important differences between the submitted version and the official published version of record. People interested in the research are advised to contact the author for the final version of the publication, or visit the $\mathrm{DOI}$ to the publisher's website.

- The final author version and the galley proof are versions of the publication after peer review.

- The final published version features the final layout of the paper including the volume, issue and page numbers.

Link to publication

\section{General rights}

Copyright and moral rights for the publications made accessible in the public portal are retained by the authors and/or other copyright owners and it is a condition of accessing publications that users recognise and abide by the legal requirements associated with these rights.

- Users may download and print one copy of any publication from the public portal for the purpose of private study or research.

- You may not further distribute the material or use it for any profit-making activity or commercial gain

- You may freely distribute the URL identifying the publication in the public portal.

If the publication is distributed under the terms of Article $25 \mathrm{fa}$ of the Dutch Copyright Act, indicated by the "Taverne" license above, please follow below link for the End User Agreement:

www.tue.nl/taverne

Take down policy

If you believe that this document breaches copyright please contact us at:

openaccess@tue.nl

providing details and we will investigate your claim. 
Exploring the electron density in plasma induced by EUV radiation: I. Experimental study in hydrogen

This content has been downloaded from IOPscience. Please scroll down to see the full text. 2016 J. Phys. D: Appl. Phys. 49145203

(http://iopscience.iop.org/0022-3727/49/14/145203)

View the table of contents for this issue, or go to the journal homepage for more

Download details:

IP Address: 131.155.151.95

This content was downloaded on 19/05/2016 at 09:15

Please note that terms and conditions apply. 


\title{
Exploring the electron density in plasma induced by EUV radiation: I. Experimental study in hydrogen
}

\author{
R M van der Horst ${ }^{1}$, J Beckers ${ }^{1}$, E A Osorio ${ }^{2}$, D I Astakhov ${ }^{3}$, \\ W J Goedheer ${ }^{4}$, C J Lee ${ }^{3}$, V V Ivanov ${ }^{5}$, V M Krivtsum ${ }^{5}$, K N Koshelev, \\ D V Lopaev ${ }^{6}$, F Bijkerk $^{3}$ and V Y Banine ${ }^{1,2}$
}

\footnotetext{
${ }^{1}$ Department of Applied Physics, Eindhoven University of Technology, PO Box 513, 5600MB Eindhoven, The Netherlands

2 ASML The Netherlands B.V., PO Box 324, 5500AH Veldhoven, The Netherlands

3 XUV Group, MESA + Institute for Nanotechnology, University of Twente, PO Box 217, 7500 AE

Enschede, The Netherlands

${ }^{4}$ FOM Institute DIFFER, PO Box 6336, 5600 HH Eindhoven, The Netherlands

5 Institute for Spectroscopy RAS (ISAN), Fizicheskaya 5, Troitsk 142190, Russian Federation

6 Skobeltsyn Institute of Nuclear Physics, Lomonosov Moscow State University, Leninskie Gory, Moscow 119991, Russian Federation
}

E-mail: r.m.v.d.horst@tue.nl

Received 8 September 2015, revised 7 December 2015

Accepted for publication 11 January 2016

Published 10 March 2016

\begin{abstract}
Plasmas induced by EUV radiation are unique since they are created without the need of any discharge. Moreover, it is essential to characterize these plasmas to understand and predict their long term impact on highly delicate optics in EUV lithography tools. In this paper we study plasmas induced by $13.5 \mathrm{~nm}$ EUV radiation in hydrogen gas. The electron density is measured temporally resolved using a non-invasive technique known as microwave cavity resonance spectroscopy. The influence of the EUV pulse energy and gas pressure on the temporal evolution of the electron density has been explored over a parameter range relevant for industry.

Our experimental results show that the maximum electron density is in the order of $10^{14} \mathrm{~m}^{-3}$ and depends linearly on the EUV pulse energy. Furthermore, the maximum electron density depends quadratically on the pressure; the linear term is caused by photoionization and the quadratic term by subsequent electron impact ionization. The decay of the plasma is governed by ambipolar diffusion and, hence, becomes slower at elevated pressures. Similarities and differences of the same processes in argon are highlighted in this paper.
\end{abstract}

Keywords: electron density, EUV, MCRS, hydrogen

(Some figures may appear in colour only in the online journal)

\section{Introduction}

Plasma is widely studied in various fields of research. However, plasma induced by EUV radiation, which is a unique kind of plasma since it is created without the need of any discharge, is scarcely investigated. These EUV-induced plasmas in hydrogen gas, in the context of EUV lithography, are the topic of this paper.
In the current generation lithography tools EUV radiation at $13.5 \mathrm{~nm}$ is used instead of UV radiation at $193 \mathrm{~nm}$. Due to the absorption of high energetic EUV photons by the background gas $(0.1-30 \mathrm{~Pa})$ in the scanner and subsequent photoionization of it, plasma is created everywhere the beam travels. The induced plasma is referred to as EUV-induced plasma. The long term impact of such a plasma on the highly delicate and expensive optics, such as the multilayer mirrors or the reticle, 


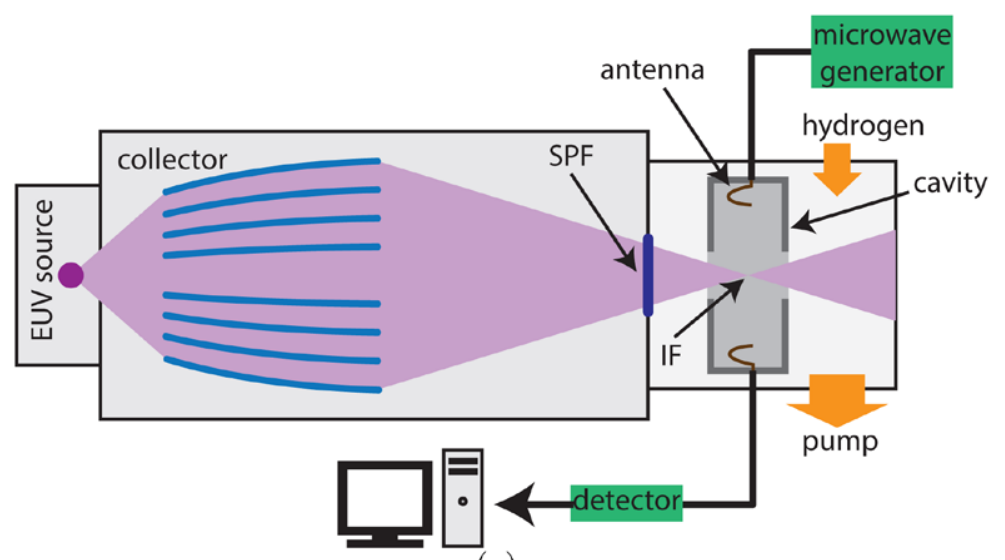

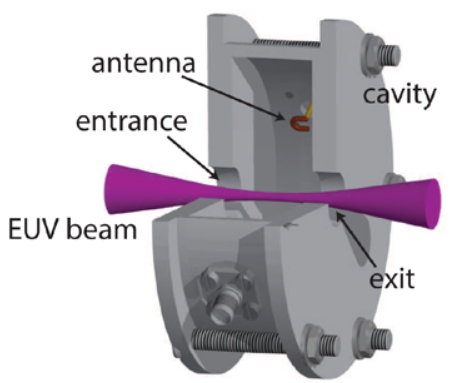

(b)

(a)

Figure 1. (a) Schematic drawing of the experimental set-up. A xenon-pinch discharge generates the EUV radiation. This radiation is focused by the collector in the intermediate focus (IF). The cavity is placed in IF. The hydrogen pressure in the measurement vessel is controlled by a needle valve [9]. (b) Drawing of the microwave cavity with an indication of the EUV beam [8].

in lithography tools is of main interest for the semiconductor industry [1]. In order to control and understand this impact, fundamental knowledge of EUV-induced plasma is essential.

Outside EUV lithography, EUV-induced plasma is also studied in the context of astrophysics. Bartnik et al studied the spectrally resolved optical emission of EUV-induced plasmas in helium and neon in the context of plasma formation in space [2-5].

Recently, Dolgov et al presented an experimental set-up to study EUV-induced processes (e.g. carbon cleaning and ion sputtering) on multilayer mirrors [6]. In order to characterize EUV-induced plasma, these authors used Langmuir probes. Previously, Van der Velden et al [1,7] studied the fundamental properties of EUV-induced plasma and its effect on the optical elements using numerical calculations. These authors also performed experiments to determine the electron density and the electron temperature using Langmuir probes. However, it was concluded in this study that Langmuir probes were not feasible to determine these parameters. Recently, we reported for the first time non-intrusive measurements of the electron density in an EUV-induced plasma in argon using a method known as microwave cavity resonance spectroscopy [8]. Moreover, in [9] we explored the influence of gas pressure and EUV pulse energy on the temporal evolution of the electron density in EUV-induced plasma in argon gas. While previously EUV lithography tools operated in low pressure argon gas, modern tools operate in low pressure hydrogen gas. Meaning that studying EUV-induced plasmas in hydrogen gas is the first step in understanding its impact on real tools. The current manuscript is part of a duo paper. In this part we focus on the experimental characterization of EUV-induced plasmas in hydrogen, while the second part focuses on modelling EUV-induced plasma in argon and hydrogen (see part II of this paper). The obtained experimental results are a first step to understanding EUV-induced plasmas in an applicational relevant configuration (similar gas and pressure). The knowledge previously obtained from experiments in argon [9] are used to interpret the experiments in hydrogen.

The goal of the research is to answer the following questions: How does the electron density in an EUV-induced hydrogen plasma depend on the gas pressure and EUV pulse energy? And how do these observations relate to experiments previously performed in argon?

\section{Experimental set-up}

The experimental set-up (see figure 1(a)) consists of three chambers, the source chamber, the collector chamber and the measurement chamber. Experimental details will be discussed below.

\subsection{EUV source}

The used xenon-based discharge produced plasma EUV source [10] is located in the source chamber. This source produces pulsed EUV radiation with a pulse duration of about $150 \mathrm{~ns}$ and a repetition rate of $500 \mathrm{~Hz}$. The radiation is collected by the collector, which is a set of water-cooled rotationally symmetric multi-shell grazing incidence mirrors, located in the collector chamber. These mirrors focus the radiation from the EUV source (first focal point) to the so-called intermediate focus (IF) in the measurement chamber. The EUV beam has a waist, which is measured with EUV sensitive foil, of $4 \mathrm{~mm}$ and a divergence of $10^{\circ} \mathrm{in} \mathrm{IF.} \mathrm{The} \mathrm{collector} \mathrm{and} \mathrm{measurement}$ chamber are differentially pumped with an aperture of $4 \mathrm{~mm}$; when the source is in operation the pressure in the collector chamber is $0.1 \mathrm{~Pa}$, while the base pressure in the measurement chamber is $10^{-4} \mathrm{~Pa}$. An SPF (spectral purity filter), which transmits between $10-20 \mathrm{~nm}$, is placed in front of the aperture.

\subsection{Measurement chamber}

The measurement chamber contains all diagnostics. The main diagnostic used was microwave cavity resonance spectroscopy (MCRS) to measure the electron density. In order to determine the EUV power, a temperature sensor was installed (as already discussed in [9]). Furthermore, the temporal shape of the EUV pulse was determined from the secondary electron emission from a copper disk. 
The spectrally integrated EUV power was determined with a temperature sensor. Since this method is extensively discussed in [9], we only discuss the key concepts here. The sensor consists of a copper disk (diameter of $25.4 \mathrm{~mm}$ and thickness of $1 \mathrm{~mm}$ ) and a temperature transducer with a repeatability of $\pm 1^{\circ}$. If the sensor is exposed to EUV radiation, its temperature will increase. After the exposure, the sensors cools down to room temperature. The heat loss coefficients are determined during the cooling phase. Using these loss coefficients, the EUV power can be determined from the temperature increase during exposure. The error in the power measurements is estimated at 5\% [9].

In order to measure the temporal shape of the EUV pulse, the temperature sensor is replaced by a copper disk which is connected to ground via a $50 \Omega$-resistor. An oscilloscope is used to measure the time resolved voltage over the resistor. The EUV radiation induces a current due to the photoelectric effect, which depends linearly on the intensity of incident radiation [11]. Hence, the temporal shape of the EUV pulse is represented by the temporal evolution of the voltage over the resistor.

\subsection{MCRS set-up}

The electron density is measured with a non-intrusive method known as microwave cavity resonance spectroscopy (MCRS). The method has already been extensively applied to study various types of plasmas in other publications [8, 9, 12-17]. Since MCRS is described in detail in these publications, we suffice with a brief summary here. In MCRS measurements the resonant frequency of a (cylindrical) resonant cavity, which depends o.a. on the electron density in the cavity, is monitored. The electron density can be calculated from the shift $\Delta \omega$ in the resonant frequency [9]:

$$
\bar{n}_{\mathrm{e}}=\frac{2 m_{\mathrm{e}} \varepsilon_{0}}{e^{2}} \frac{\omega^{2}}{\omega_{0}} \Delta \omega
$$

with $m_{\mathrm{e}}$ the electron mass, $e$ the elementary charge and $\omega_{0}$ and $\omega$ the resonant frequencies without and with plasma, respectively. It should be noted that MCRS gives the average electron density weighted by the square of the local electric field of the excited resonant mode $\vec{E}(\vec{r})$ [9]:

$$
\bar{n}_{\mathrm{e}}=\frac{\int_{\text {cavity }} n_{\mathrm{e}}(\vec{r}) E^{2}(\vec{r}) \mathrm{d} \vec{r}}{\int_{\text {cavity }} E^{2}(\vec{r}) \mathrm{d} \vec{r}} .
$$

The aluminium microwave cavity, which has an inner radius of $33 \mathrm{~mm}$ and an inner height of $20 \mathrm{~mm}$, is positioned around the intermediate focus. In order for the EUV beam to pass through the cavity, holes of $13 \mathrm{~mm}$ are drilled in the top and bottom plates. Inside the cavity, two copper antennas are mounted to the side walls opposite to each other; these antennas are not subject to EUV irradiation. One of the antennas is connected to a microwave generator (Stanford Research Systems SG386) to excite the $\mathrm{TM}_{010}$-mode at $3.49674 \pm 0.00004 \mathrm{GHz}$. The other antenna is connected to a microwave detector (Hittite HMC602LP4), which has a time resolution of $10 \mathrm{~ns}$. The output of the detector is recorded by a transient recorder (Spectrum M3i.4121-exp). The resonant

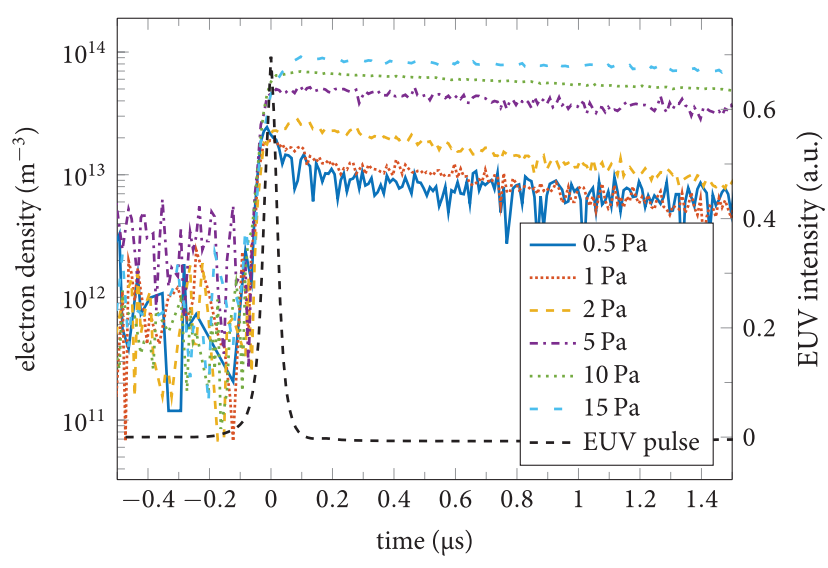

Figure 2. Square-electric-field-weighted average electron density as a function of time for various hydrogen pressures during and shortly after plasma ignition. The black dashed line shows the EUV intensity. The EUV pulse energy is $(44 \pm 2) \mu \mathrm{J}$.

frequency is determined by sweeping the frequency of the microwave generator over a predefined range and measuring the response of the detector using the transient recorder. At every point in time, the response is fitted as a function of the frequency with a (two component) Fourier series. The measurements are performed with in-house developed software. In [8] it was shown that the time resolution of the MCRS set-up is $17 \mathrm{~ns}$ and that the error in the square-electric-fieldweighted average electron density is less than $30 \%$ with a detection limit of $2 \times 10^{12} \mathrm{~m}^{-3}$.

\section{Results}

\subsection{Gas pressure}

The square-electric-field-weighted average electron density $\left(\bar{n}_{\mathrm{e}}\right)$ is measured as a function of time for various hydrogen pressures in the range from 0.5 to $15 \mathrm{~Pa}$. Figures 2 and 3 show the results for an EUV pulse energy of $44 \mu \mathrm{J}$ on short and long time scales respectively in order to show both the relatively fast increase of the electron density and the slower decay. These results show that $\bar{n}_{\mathrm{e}}$ increases with pressure above $1 \mathrm{~Pa}$, while below $1 \mathrm{~Pa}$ the electron density appears to be independent of pressure. The maximum $\bar{n}_{\mathrm{e}}$ is reached almost immediately after the EUV pulse. Furthermore, the results show that the decay time increases if the pressure increases. The signalto-noise ratio becomes very small below $10^{13} \mathrm{~m}^{-3}$, since the detection limit of our diagnostics $\left(\sim 10^{12} \mathrm{~m}^{-3}\right)$ is approached.

The maximum square-electric-field-weighted average electron density $\left(\bar{n}_{\mathrm{e}}^{\max }\right)$ as a function of pressure is plotted in figure 4. A quadratic fit could be nicely fitted through the experimental data points, as will be explained in section 4.1. The quadratic fit has the following expression: $\bar{n}_{\mathrm{e}}^{\max }=(3 \pm 4) \times 10^{11} p^{2}+(5 \pm 4) \times 10^{12} p+(1.8 \pm 0.6) \times 10^{13}$.

\subsection{EUV pulse energy}

The maximum square-electric-field-weighted average electron density is measured as a function of the EUV pulse 


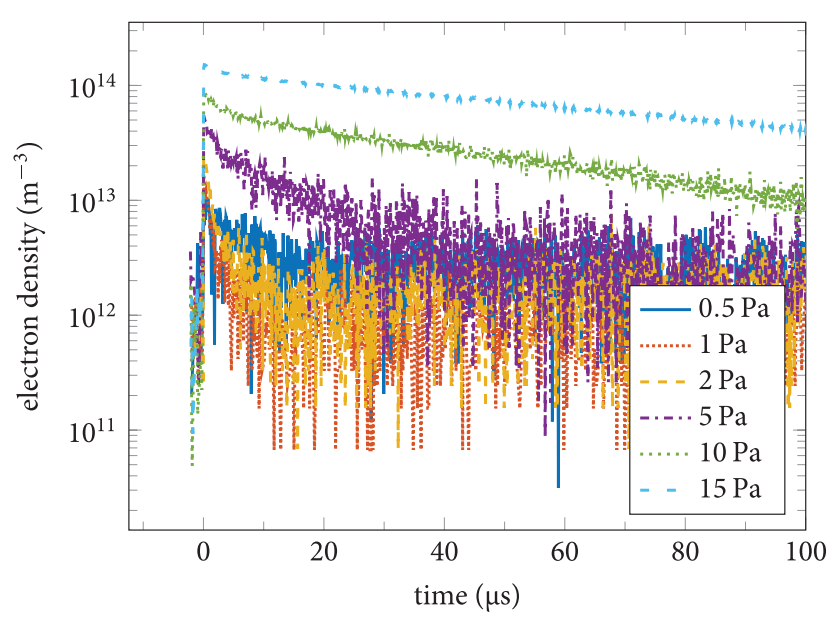

Figure 3. Square-electric-field-weighted average electron density as a function of time for various hydrogen pressures at longer time scales. The energy of the EUV pulse is $(44 \pm 2) \mu \mathrm{J}$.

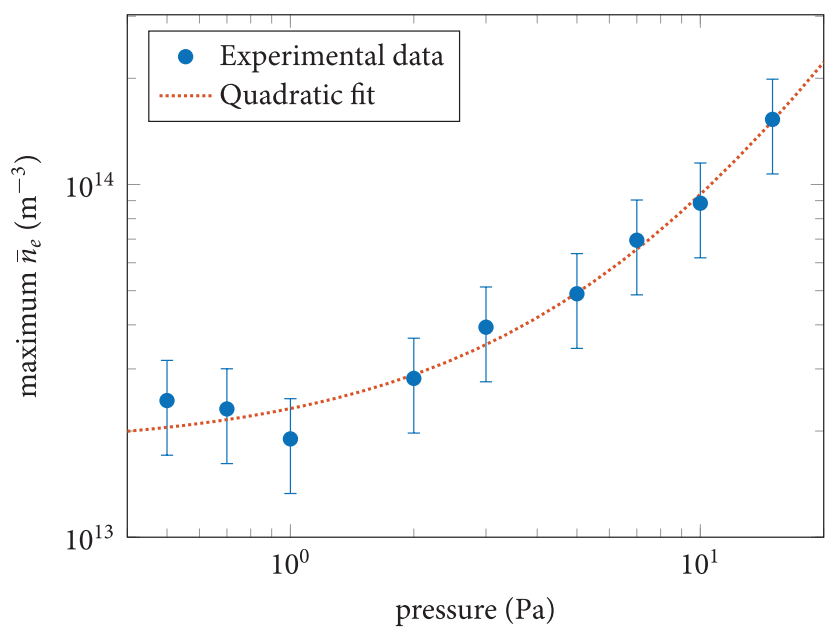

Figure 4. Maximum $\bar{n}_{\mathrm{e}}$ as a function of gas pressure for an EUV pulse energy of $(44 \pm 2) \mu \mathrm{J}$. The expression of the quadratic fit is $\bar{n}_{\mathrm{e}}^{\max }=(3 \pm 4) \times 10^{11} p^{2}+(5 \pm 4) \times 10^{12} p+(1.8 \pm 0.6) \times 10^{13}$.

energy as well. The results at a pressure of 4.6 Pa are shown in figure 5 . The data could be nicely fitted with a linear fit with an intercept fixed at zero (no photons, no photoionization): $\bar{n}_{\mathrm{e}}^{\max }=(1.1 \pm 0.2) \times 10^{12} E_{\mathrm{EUV}}$.

\section{Discussion and interpretation}

The square-electric-field-weighted average electron density has been measured as a function of hydrogen pressure and EUV pulse energy. In this section, the relevant physical processes governing EUV-induced hydrogen plasma will be discussed and the obtained results will be compared to experiments performed in argon [9].

\subsection{Influence of gas pressure on the maximum electron density}

First, the creation of the EUV-induced plasma in hydrogen is discussed. As already discussed in detail in our previous

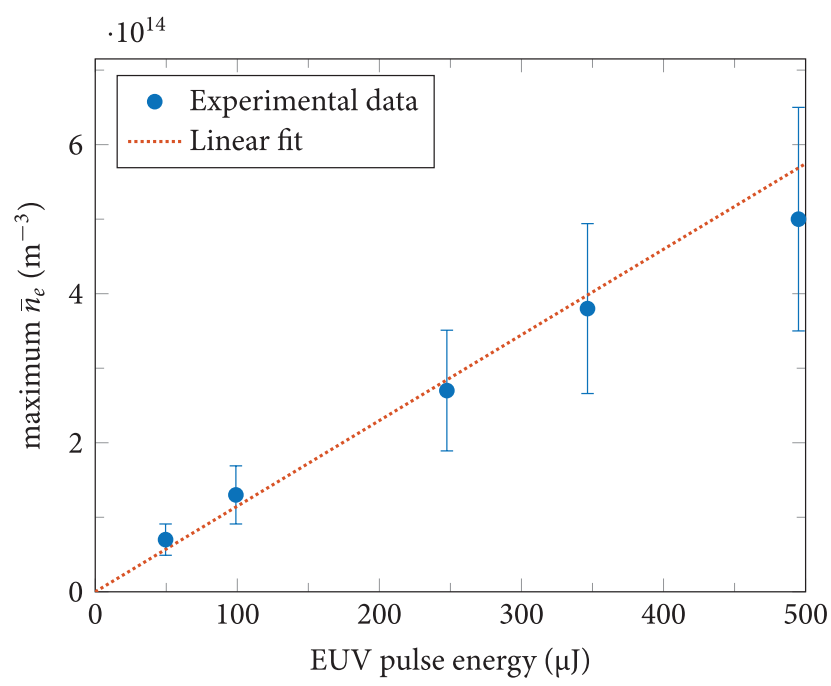

Figure 5. Maximum $\bar{n}_{\mathrm{e}}$ as a function of EUV pulse energy at a pressure of 4.6 Pa. The fit has a slope of $(1.1 \pm 0.2) \times 10^{12} \mathrm{~m}^{-3} \mu \mathrm{J}^{-1}$ and the intercept was fixed at zero.

publication [9], the temporal evolution of $\bar{n}_{\mathrm{e}}$ is governed by the following production and loss processes:

(i) Production due to photoionization

(ii) Production due to electron impact ionization

(iii) Loss of initial high energy electrons $L_{\mathrm{fe}}$

(iv) Apparent loss due to averaging $L_{\mathrm{av}}$

(v) Loss due to recombination $L_{\text {rec }}$

Next, these processes are described briefly.

- Production due to photoionization. The first production process is production of electrons by absorption of EUV photons $\gamma$ and subsequent ionization $P_{\text {pi }}$. Basically three processes may occur when a photon is absorbed by a hydrogen molecule: single photoionization (equation (3)), dissociative photoionization (equation (4)) and double photoionization (equation (5)):

$$
\begin{gathered}
\gamma+\mathrm{H}_{2} \rightarrow \mathrm{e}^{-}+\mathrm{H}_{2}^{+}(X) \\
\gamma+\mathrm{H}_{2} \rightarrow \mathrm{e}^{-}+\mathrm{H}_{2}^{+*} \rightarrow \mathrm{e}^{-}+\mathrm{H}^{*}+\mathrm{H}^{+} \\
\gamma+\mathrm{H}_{2} \rightarrow 2 \mathrm{e}^{-}+\mathrm{H}_{2}^{2+} \rightarrow 2 \mathrm{e}^{-}+2 \mathrm{H}^{+},
\end{gathered}
$$

where $X$ denotes the ground state; the single ionization energy of hydrogen is $15.4 \mathrm{eV}$ [18], while the double ionization energy is $47-48 \mathrm{eV}$ [19]. The cross section at $92 \mathrm{eV}$ are $4.8 \times 10^{-24} \mathrm{~m}^{2}, 1.0 \times 10^{-24} \mathrm{~m}^{2}$ and $2.8 \times 10^{-25} \mathrm{~m}^{2}$ respectively [19-21]. The first reaction contributes to about $81 \%$ of the ionization events, the second to about $15 \%$ and the third to about $5 \%$ (for a photon of $92 \mathrm{eV}$ ), meaning that on average 1.05 electrons are generated per absorbed photon. Hence, mainly the $\mathrm{H}_{2}^{+}$ion will be created. However, this ion is rapidly converted to $\mathrm{H}_{3}^{+}$in collision with $\mathrm{H}_{2}\left(\mathrm{H}_{2}^{+}+\mathrm{H}_{2} \rightarrow \mathrm{H}_{3}^{+}+\mathrm{H}\right)$ within a microsecond [22-24]. This means that the dominant ion in a hydrogen plasma will be $\mathrm{H}_{3}^{+}$. Due to momentum conservation, almost all excess energy (77 eV for equation (3)) 
is transferred to the electrons, while the ions remain at roughly room temperature.

- Production due to electron impact ionization. The second production process is production of electrons by electron impact ionization $P_{\mathrm{ei}}$ :

$$
\begin{gathered}
\mathrm{e}^{-}+\mathrm{H}_{2} \rightarrow 2 \mathrm{e}^{-}+\mathrm{H}_{2}^{+}(X) \\
\mathrm{e}^{-}+\mathrm{H}_{2} \rightarrow 2 \mathrm{e}^{-}+\mathrm{H}+\mathrm{H}^{+},
\end{gathered}
$$

with cross section at $77 \mathrm{eV}$ of $8.7 \times 10^{-21} \mathrm{~m}^{2}$ and $7.5 \times 10^{-21} \mathrm{~m}^{2}$ respectively [25]. Hence, the first reaction contributes to $92 \%$ and the second to $8 \%$ of the ionization events.

- Loss of high energy electrons. The initial high energy electrons generated by photoionization escape from the centre of the cavity to the wall, leaving the positive ions behind. As a result a potential difference is induced between the plasma and the cavity wall, confining the remaining electrons. In [9] we estimated the charge density needed to confine the plasma using a model of a coaxial cable. The square-electric-field-weighted average space charge needed to confine the electrons was found to be $6 \times 10^{12} \mathrm{~m}^{-3}$. Since the average ion charge is 1.05 , this corresponds to an $\bar{n}_{\mathrm{e}}$ of $6 \times 10^{12} \mathrm{~m}^{-3}$, as to be measured with the MCRS method. This means that, opposite to the argon case [9], the loss term due to fast electrons cannot be neglected in the case of hydrogen since the typical $\bar{n}_{\mathrm{e}}$ in EUV-induced hydrogen plasma is $10^{14} \mathrm{~m}^{-3}$ here. Hence, the measured maximum $\bar{n}_{\mathrm{e}}$ will always be underestimated by a constant value of about $6 \times 10^{12} \mathrm{~m}^{-3}$.

- Apparent loss due to averaging. The second loss process-which is an apparent one-is related to the square-electric-field-weighted averaging $L_{\mathrm{av}}$. In the case of argon, we showed that during the first microsecond $\bar{n}_{\mathrm{e}}$ was constant, meaning that the apparent loss due to averaging could be neglected [9]. The expansion of plasma in hydrogen gas is much faster than in argon because of the lower ion inertia, which is why $\bar{n}_{\mathrm{e}}$ cannot be considered constant during the first $\mu$ s as was the case in argon gas. Since the expansion speed of plasma in hydrogen gas is less than 10 times faster than in argon gas [23, 26], it is assumed that the effect of averaging is still negligible during the first $100 \mathrm{~ms}$. Hence, if $\bar{n}_{\mathrm{e}}^{\max }$ occurs at time scales shorter than 100 ns after EUV irradiation, this value has not been influenced by averaging.

- Loss due to recombination. Since the time needed for the plasma to reach the wall (radius of $33 \mathrm{~mm}$ ) is even longer than the previously mentioned $100 \mathrm{~ns}$, losses due to wall recombination $L_{\text {rec }}$ are consequently negligible at time scales shorter than $100 \mathrm{~ns}$.

The typical time scales are summarized in table 1. During the first $100 \mathrm{~ns}$ after EUV irradiation, the (apparent) loss processes due to averaging and recombination are negligible. Hence, only production processes and loss of high energy electrons need to be taken into account.

Figure 2 shows that the maximum $\bar{n}_{\mathrm{e}}$ does not vary with pressure below $1 \mathrm{~Pa}$. This means that there should be a
Table 1. Characteristic time scales for various processes in EUVinduced plasmas in hydrogen.

\begin{tabular}{ll}
\hline Process & Typical time scale \\
\hline EUV pulse length & $100 \mathrm{~ns}$ \\
Post EUV ionization time & $0.1-1 \mu \mathrm{s}$ \\
Time to reach wall & $1-10 \mu \mathrm{s}$ \\
Total decay time & $1-100 \mu \mathrm{s}$ \\
Time between EUV pulses & $2 \mathrm{~ms}$ \\
\hline
\end{tabular}

pressure independent source of electrons, which could for instance be secondary electrons generated from nearby walls by the photoelectric effect.

The maximum $\bar{n}_{\mathrm{e}}\left(\bar{n}_{\mathrm{e}}^{\max }\right)$ is reached before $100 \mathrm{~ns}$, meaning that the rate equation for the electron density includes the production processes and the loss of high energy electrons only. It was derived in [9] that the production of electrons by photoionization and subsequent electron impact ionization in argon is given by:

$$
n_{\mathrm{e}}^{\max }=\frac{1.2 \sigma_{\mathrm{pi}} n_{\mathrm{a}} E_{\mathrm{EUV}}}{A_{\mathrm{EUV}} E_{\mathrm{ph}}}\left[1+k_{\mathrm{e} i} n_{\mathrm{a}}\left(t_{\mathrm{m}}-\frac{1}{2} \tau\right)\right] .
$$

where $\sigma_{\mathrm{pi}}$ is the photoionization cross section, $n_{\mathrm{a}}$ is the background density, $A_{\mathrm{EUV}}$ is the cross section of the EUV beam, $E_{\mathrm{ph}}$ is the photon energy, $\tau$ is the duration of the EUV pulse, $t_{\max }$ is the time at which the maximum $\bar{n}_{\mathrm{e}}$ is reached, $k_{\mathrm{ei}}$ is the electron impact ionization rate and $E_{\mathrm{EUV}}$ is the EUV pulse energy. This equation also hold for hydrogen, if the factor 1.2 , which is the average number of electrons generated per absorbed photon in argon, is replaced by the corresponding factor in hydrogen, which is 1.05 . Above, it was concluded that also a pressure independent production term $P\left(E_{\mathrm{EUV}}\right)$ and the loss of high energy electrons $L_{\mathrm{fe}}$ need to be taken into account. Hence, the maximum electron density is:

$$
\begin{aligned}
n_{\mathrm{e}}^{\max }= & \frac{1.05 \sigma_{\mathrm{pi}} n_{\mathrm{gas}} I_{0} \tau}{E_{\mathrm{ph}}}\left(1+\bar{k}_{\mathrm{ei}} n_{\mathrm{gas}}\left(t_{m}-\frac{1}{2} \tau\right)\right) \\
& +P\left(E_{\mathrm{EUV}}\right)-L_{\mathrm{fe}},
\end{aligned}
$$

The maximum $\bar{n}_{\mathrm{e}}$ should quadratically depend on the pressure, which is close to what is observed in the experiments; a quadratic function could be fitted through the experimental data points (see figure 4). It should be noted that the quadratic increase of the maximum electron density with pressure is limited, since the maximum contribution by electron impact ionization is limited to about 5 ionizations, i.e. the initial electron energy after photoionization divided by the ionization energy of hydrogen, per initially absorbed photon.

The constant term $\left((1.8 \pm 0.6) \times 10^{13} \mathrm{~m}^{-3}\right)$ of the quadratic fit in figure 4 is related to the loss of high energy electrons $L_{\mathrm{fe}}$ and the pressure independent source $P\left(E_{\mathrm{EUV}}\right)$. Since the sum of both processes is positive, the source is dominant. In the argon case [9], the constant contribution-which should be in the same order of magnitude- to the maximum $\bar{n}_{\mathrm{e}}$ was not observed, since it was negligible compared to $\bar{n}_{\mathrm{e}}^{\max }\left(10^{15} \mathrm{~m}^{-3}\right)$. However, since the maximum $\bar{n}_{\mathrm{e}}$ in hydrogen is significantly lower, this contribution is more pronounced in hydrogen. 
The linear part of the quadric fit in figure 4 is related to photoionization and has a coefficient of $(5 \pm 4) \times 10^{12} \mathrm{~m}^{-3} \mathrm{~Pa}^{-1}$. To convert the measured coefficient to a coefficient with is valid for the electron density in the centre of the cavity, a spatial distribution of the electron density is assumed; it is assumed that this distribution is a square with a width equal to the diameter of the EUV beam, i.e. $4 \mathrm{~mm}$. Since the mode of the resonance cavity is known $\left(\mathrm{TM}_{010}\right.$-mode $), \bar{n}_{\mathrm{e}}$ is converted to an electron density in the centre of the cavity using equation (2). This results in a linear coefficient of $4.7 \times 10^{14} \mathrm{~m}^{-3} \mathrm{~Pa}^{-1}$, which is valid in the centre of the cavity. These coefficients correspond to the linear term in equation (9):

$$
\alpha_{t}=\frac{1.05 \sigma_{\mathrm{pi}} n_{\mathrm{a}} E_{\mathrm{EUV}}}{A_{\mathrm{EUV}} E_{\mathrm{ph}}} \frac{1}{p},
$$

which is rewritten using the ideal gas law to:

$$
\alpha_{t}=\frac{1.05 \sigma_{\mathrm{pi}} E_{\mathrm{EUV}}}{A_{\mathrm{EUV}} E_{\mathrm{ph}} k_{\mathrm{B}} T_{\mathrm{g}}},
$$

where $p$ is the gas pressure, $k_{\mathrm{B}}$ is the Boltzmann constant and $T_{\mathrm{g}}$ is the gas temperature. Since the cross section, photon energy and EUV pulse energy depend on the wavelength of the photon $\lambda$, the previous equation needs to be rewritten to:

$$
\alpha_{t}=\int_{0}^{\infty} \frac{1.05 \sigma_{\mathrm{pi}}(\lambda) E_{\mathrm{EUV}}(\lambda)}{k_{\mathrm{B}} T_{\mathrm{g}} A_{\mathrm{EUV}} h c / \lambda} \mathrm{d} \lambda,
$$

To calculate this coefficient theoretically, the total photoionization cross section and the spectrally resolved EUV pulse energy are needed. The cross sections are taken from [19-21]. The relative spectrum of the EUV source as used here was measured by Kieft [27]. To obtain the spectrum of the light irradiating the gas inside the cavity, the spectrum of the EUV source is multiplied by the transmission of the collector and the used SPF filter. The resulting relative spectrum was then calibrated with a wavelength integrated pulse energy measurement of the EUV source (here $(44 \pm 2) \mu \mathrm{J}$ per pulse). This results in a theoretical linear coefficient of $1 \times 10^{15} \mathrm{~m}^{-3} \mathrm{~Pa}^{-1}$, which corresponds well with the experimental value $\left(4.7 \times 10^{14} \mathrm{~m}^{-3} \mathrm{~Pa}^{-1}\right)$.

The quadratic term is related to electron impact ionization. Since the cross section for this process is highly dependent on the electron energy, which is an unknown parameter during the first $100 \mathrm{~ns}$, it is not possible to give a reasonable theoretical estimate of the coefficient. However, using equation (9) the average electron impact ionization rate $\bar{k}_{\mathrm{ei}}$ can be estimated from the experimentally obtained quadratic coefficient. This yields a rate $\bar{k}_{\mathrm{ei}} \approx 10^{-16} \mathrm{~m}^{3} \mathrm{~s}^{-1}$, which is a reasonable rate for an electron temperature of a few electronvolts $\left(10^{-18}-10^{-15} \mathrm{~m}^{3} \mathrm{~s}^{-1}\right.$ between $2-7 \mathrm{eV}$ ) [25].

In conclusion, during the first 100 ns the loss of fast electrons cannot be neglected in hydrogen. The maximum electron density depends quadratically on the gas pressure. In the quadratic fit describing the pressure dependence of the maximum $\bar{n}_{\mathrm{e}}$, the constant term is most probably due to secondary electrons and the loss of fast electrons, the linear term is due to photoionization and the quadratic term is due to electron impact ionization.
4.2. Influence of EUV pulse energy on the maximum electron density

The maximum $\bar{n}_{\mathrm{e}}$ as a function of EUV pulse energy was studied as well (figure 5). As expected (equation (9)), the maximum $\bar{n}_{\mathrm{e}}$ depends linearly on the EUV pulse energy. The slope of a linear fit through the experimental data points is $(1.1 \pm 0.2) \times 10^{12} \mathrm{~m}^{-3} \mu \mathrm{J}^{-1}$.

\subsection{Decay of EUV-induced plasma}

At a certain moment, typically $100 \mathrm{~ns}$ after EUV irradiation, loss processes start to dominate and the plasma starts to decay (figure 3). As mentioned before, due to the escape of initial electrons generated by photoionization, a potential difference is induced between the plasma and the wall. The potential difference causes an ambipolar flow [28]. First, the plasma expands towards the cavity walls. During this period the electron density as measured with MCRS decreases, due to the decrease in the weighting factor of the MCRS technique closer to the wall (lower electric field of the excited mode closer to the walls). This, however, does not imply that the total number of electrons also decreases. The speed of the expansion to the wall is typically limited by the ion acoustic speed $\left(\sqrt{k_{\mathrm{B}} T_{\mathrm{e}} / m_{\mathrm{i}}}\right)$, which is about $v=2000-20000 \mathrm{~m} \mathrm{~s}^{-1}$ for an electron temperature of $0.1-10 \mathrm{eV}$ [29]. Thus, after about 2-20 $\mu$ s the plasma has reached the wall of the cavity. From this moment on, the electrons and ions recombine at the wall, meaning that the decay is governed by ambipolar diffusion and subsequent wall recombination. The typical time scale of this process is [30]:

$$
\tau=\left[\left(\frac{2.405}{R}\right)^{2}+\left(\frac{\pi}{H}\right)^{2}\right]^{-1} \frac{1}{D_{a}},
$$

with $R$ and $H$ the radius and height of the cavity and $D_{a}$ the ambipolar diffusion coefficient. Equation (13) is only valid if an ambipolar equilibrium has been established. The ambipolar diffusion coefficient depends on the ion mobility $\mu_{\mathrm{i}}$, the electron temperature $\hat{T}_{\mathrm{e}}$ and ion temperature $\hat{T}_{\mathrm{i}}$ (in $\mathrm{eV}$ ):

$$
D_{a}=\mu_{\mathrm{i}}\left(\hat{T}_{\mathrm{e}}+\hat{T}_{\mathrm{i}}\right)
$$

Since the ion mobility decreases with pressure [31], the decay time increases with pressure. This explains the observation that the plasma decays slower at elevated pressures.

Volume recombination due to two- or three-body recombination can be neglected, since the typical time scales for these processes (10 $\mu \mathrm{s}$ [32]) is much slower than the observed decay times (tens of microseconds).

\subsection{Comparison of EUV-induced plasma in argon and hydrogen}

The physical processes governing EUV-induced plasmas in argon and hydrogen are very similar. However, due to the different properties of both gases the measured $\bar{n}_{\mathrm{e}}$ and time scales are very different. 
The production due to photoionization is $(5 \pm 4) \times 10^{12}$ $\mathrm{m}^{-3} \mathrm{~Pa}^{-1}$ in hydrogen, which is about a factor 24 lower than the value found from experiments in argon [9] (corrected for the difference in EUV pulse energy). This, however, is what one should expect, considering that the photoionization cross section is a factor 23 lower [19-21, 33].

The slope of $\bar{n}_{\mathrm{e}}^{\mathrm{max}}$ as a function of the EUV pulse energy in hydrogen is a factor $17 \pm 4$ lower than in the case of argon [9], which is less pronounced than expected from the lower photoionization cross section (factor 23). The reason for the discrepancy is most probably the fact that the EUV pulse energy was measured differently during this measurement campaign. Instead of the temperature sensor described in section 2, the EUV pulse energy was determined from the discolouration of an EUV sensitive foil. This method is less accurate than the temperature sensor.

The observed decay times of the induced plasma in hydrogen gas are a factor 7-10 faster than the decay times observed in argon gas [9]. This is also what is expected from the difference in ion mobility (factor $7.3[23,26]$ ).

\section{Conclusions}

The electron density is studied in pulsed EUV-induced plasmas in hydrogen. It was found that the maximum squareelectric-field-weighted average electron density increases quadratically with hydrogen pressure, which is expected from theoretical considerations and which is also observed in the case of argon [9]. The linear part of the quadratic dependence is due to photoionization, while the quadratic term is due to subsequent electron impact ionization. The experimental values correspond well with theoretical estimations, which means that the theoretical model yields accurate predictions of the maximum electron density. Furthermore, the experiments showed that $\bar{n}_{\mathrm{e}}$ depends linearly on the EUV pulse energy. This is also expected from theory and what is observed in the case of argon. The decay of $\bar{n}_{\mathrm{e}}$ is governed by ambipolar diffusion and subsequent wall recombination. At elevated pressures, the decay time becomes longer because of the decrease in ion mobility.

Previously, similar experiments have been conducted in EUV-induced plasmas in argon [9]. In both argon and hydrogen, similar dependencies have been found, meaning that the physical processes governing EUV-induced plasma are also similar. However, the measured electron densities in argon were significantly higher than in hydrogen due to the higher photoionization cross section. Furthermore, the time scale of plasma decay is longer in argon than in hydrogen (factor 10) due to the increased ion mobility (factor 7)

Although the impact of EUV-induced plasma on multilayer mirrors (MLMs) or the reticle cannot be fully assessed yet, some trends can be deduced from the obtained results. With increasing pressure and EUV pulse energy, the electron density and consequently the ion density increases. This will result in a larger impact of the EUV-induced plasma on the optical elements. In order to assess the full impact, the influence of the MLMs or the reticle on the EUV-induced plasma and vice versa need to be studied as well.

\section{Acknowledgments}

The authors would like to acknowledge ASML for financial support and the opportunity to use its EUV sources.

\section{References}

[1] van der Velden M, Brok W, van der Mullen J, Goedheer W and Banine V 2006 Phys. Rev. E 73036406

[2] Bartnik A, Fedosejevs R, Wachulak P, Fiedorowicz H, Serbanescu C, Saiz E, Riley D, Toleikis S and Neely D 2013 Laser Part. Beams 31195

[3] Bartnik A, Wachulak P, Fiedorowicz H, Jarocki R, Kostecki J and Szczurek M 2013 Radiat. Phys. Chem. 939

[4] Bartnik A, Fiedorowicz H and Wachulak P 2014 Phys. Plasmas 21073303

[5] Bartnik A, Wachulak P, Fiedorowicz H, Fok T, Jarocki R and Szczurek M 2014 Phys. Scr. T161 014061

[6] Dolgov A, Yakushev O, Abrikosov A, Snegirev E, Krivtsun V. M, Lee C J and Bijkerk F 2015 Plasma Sources Sci. Technol. 24035003

[7] van der Velden M 2008 Phd Thesis Eindhoven University of Technology, Eindhoven (http://alexandria.tue.nl/ extra2/200810574.pdf)

[8] van der Horst R M, Beckers J, Nijdam S and Kroesen G M W 2014 J. Phys. D: Appl. Phys. 47302001

[9] van der Horst R M, Beckers J, Osorio E A and Banine V Y 2015 J. Phys. D: Appl. Phys. 48285203

[10] Bergmann K, Schriever G, Rosier O, Müller M, Neff W and Lebert R 1999 Appl. Opt. 385413

[11] Young H D and Freedman R A 2004 University Physics 11th edn (Reading, MA: Addison-Wesley)

[12] Rose D J and Brown S C 1952 J. Appl. Phys. 231028

[13] Agdur B and Enander B 1962 J. Appl. Phys. 33575

[14] Stoffels E, Stoffels W, Vender D, Kando M, Kroesen G and de Hoog F 1995 Phys. Rev. E 512425

[15] Beckers J, Stoffels W W and Kroesen G M W 2009 J. Phys. D: Appl. Phys. 42155206

[16] Gundermann S, Loffhagen D, Wagner H E and Winkler R 2001 Contrib. Plasma Phys. 4145

[17] van de Wetering F M J H, Beckers J and Kroesen G M W 2012 J. Phys. D: Appl. Phys. $\mathbf{4 5} 485205$

[18] Lias S 2015 NIST Chemistry WebBook, NIST Standard Reference Database Number 69 ed W G Mallard and P G Linstrom (Gaithersburg: National Institute of Standards and Technology)

[19] Chung Y M, Lee E M, Masuoka T and Samson J A R 1993 J. Chem. Phys. 99885

[20] Dujardin G, Besnard M, Hellner L and Malinovitch Y 1987 Phys. Rev. A 355012

[21] Kossmann H, Schwarzkopf O, Kammerling B, Braun W and Schmidt V 1989 J. Phys. B: At. Mol. Opt. Phys. 22 L411

[22] Cosby P and Helm H 1988 Chem. Phys. Lett. 15271

[23] Albritton D, Miller T, Martin D and McDaniel E 1968 Phys. Rev. 17194

[24] Oka T 2013 Chem. Rev. 1138738

[25] Yoon J S, Song M Y, Han J M, Hwang S H, Chang W S, Lee B and Itikawa Y 2008 J. Phys. Chem. Ref. Data 37913 
[26] Helm H and Elford M T 1977 J. Phys. B: At. Mol. Phys. 103849

[27] Kieft E R 2008 private communications with ASML research

[28] Franklin R N 2003 J. Phys. D: Appl. Phys. 36828

[29] Samir U, Wright K. H and Stone N H 1983 Rev. Geophys. 211631

[30] Luikov A 1968 Analytical Heat Diffusion Theory (New York: Academic)
[31] Ellis H, Pai R, McDaniel E, Mason E and Viehland L 1976 At. Data Nucl. Data Tables 17177

[32] Glosik J, Korolov I, Plasil R, Novotny O, Kotrik T, Hlavenka P, Varju J, Mikhailov I A, Kokoouline V and Greene C H 2008 J. Phys. B: At. Mol. Opt. Phys. 41191001

[33] Saito N and Suzuki I H 1992 Int. J. Mass Spectrom. Ion Process. 115157 